\title{
Nucleation of twins and dislocations in V-Ti alloys under various straining conditions
}

\author{
Aleksandr Korchuganov ${ }^{1, *}$, Dmitrij Kryzhevich ${ }^{1}$, and Konstantin Zolnikov ${ }^{1}$ \\ ${ }^{1}$ Institute of Strength Physics and Materials Science, Siberian Branch, Russian Academy of Sciences, \\ 634055 Tomsk, Russian Federation
}

\begin{abstract}
The features of plasticity nucleation in V-4Ti and V-16Ti crystallites under uniaxial tension and bilateral compression are studied. It is shown that the nucleation of plasticity in crystallites is associated with the formation of twins under uniaxial tension. During the development of plasticity, a screw dislocation cell structure is formed between twin plates. The strain and stress at which plasticity nucleates in the material decreases with increasing Ti concentration. It was found that the distribution pattern of Ti atoms in the initial structure has a significant effect on the elastic limit of the simulated crystallites. The plastic deformation of crystallites with $16 \%$ Ti under bilateral compression is realized only by the dislocation mechanism. This behavior of the material is due to the low value of the stress at the elastic limit, which is insufficient for the formation of twins.
\end{abstract}

\section{Introduction}

Along with low-activation ferritic-martensitic steels [1-3], vanadium-based alloys are promising structural materials in the nuclear power industry $[4,5]$. They demonstrate high superplasticity, thermal conductivity, excellent resistance to swelling and low activation under irradiation. Experimental data indicates that titanium is one of the key additives to vanadium-based alloys that improve their performance characteristics. The elastic and strength properties of V-Ti alloys have been rather well studied for low rates of mechanical loading. At present, the mechanical properties and features of the structural defect nucleation in materials under high-rate loading are the subject of active computer simulation studies [6-11]. Computer simulation overcomes the difficulties that arise at the experimental studying of the dynamics of structural changes in the material at the micro level. The molecular dynamics method made it possible to study in detail the behavior of materials at the atomic level, taking into account their stoichiometric composition and internal structure under various types of loading [12-16]. The greatest success in studying the properties and behavior of metals and alloys was achieved for materials with an fcc lattice [17-19]. The delay in the study of materials with a bcc lattice is largely due to the lack of reliable and effective potentials for simulation of their behavior at the atomic level.

As a rule, plastic deformation in bcc materials is due to the dislocation glide and twinning $[20,21]$. It is known that during high-rate deformation, a material with a bcc

\footnotetext{
* Corresponding author: avkor@ispms.ru
} 
lattice can behave like a viscous liquid, preserving the crystal lattice, high strength and hardness [12]. The most studied among metals with a bcc lattice is iron. It was shown in [22] that dislocation slip is the main mechanism of plasticity in single crystals of bec iron at room temperature and low strain rates. With a decrease in temperature and/or an increase in the strain rate, the contribution of the twinning process to the development of plasticity of a single crystal increases. The orientation of the single crystal relative to the loading directions has a significant effect on twinning [22, 23]. When stretching along the directions close to the [001] crystallographic axis, the single crystal of iron is deformed on the basis of twinning, and when stretched along the directions close to the [110] and [111] axes, the twinning is significantly suppressed. The results of simulation of the behavior of nanoscale iron single crystals are undoubtedly useful in studying V-Ti alloys both in terms of similarity and differences in the behavior of materials with a bcc structure. It should be taken into account that the properties and peculiarities of plasticity nucleation differ significantly for nanoscale crystallites and bulk single crystals [24, 25]. This is due to the increasing contribution from free surfaces to the deformation process. As a rule, for the nucleation of plasticity in nanocrystals, it is necessary to achieve stresses close to theoretical strength $[25,26]$. The results of computer simulation of the deformation behavior of nanoscale crystallites are also of considerable interest for the study of nanocrystalline materials [23, 27-29].

In this paper, the mechanisms of plasticity nucleation in nanoscale bcc V-Ti single crystals with a different stoichiometric composition for various schemes of high-rate mechanical loading have been studied on the basis of molecular dynamics simulation.

\section{Methods}

Molecular dynamics calculations were performed in the LAMMPS [30] package. The interatomic interaction in the V-Ti alloy was described by a many-body potential [31] developed in the framework of the embedded atom method. V-4Ti and V-16Ti samples were simulated with a random distribution of $\mathrm{Ti}$ atoms (samples of the first type) and samples after minimizing energy by rearranging Ti atoms using the Metropolis Monte Carlo algorithm (samples of the second type). The relaxation scheme for samples of the second type is described in [32], and the algorithm itself in [33]. The initial crystallite sizes along [ $11 \overline{2}],[111]$ and $[1 \overline{1} 0]$ directions were $45 \times 30 \times 30 \mathrm{~nm}$. Periodic boundary conditions were used in all directions. The temperature of the samples during the whole calculation was maintained equal to $300 \mathrm{~K}$. Mechanical loading of crystallites was carried out according to two schemes: 1) uniaxial tension — the crystallites were stretched along the [11 direction, and in [111] and [1 $1 \overline{1} 0$ ] directions zero stresses were maintained; 2) loading in constrained conditions - the samples were compressed along [111] and [1 $\overline{1} 0]$ directions, and zero stress was maintained along the $[11 \overline{2}]$ direction. The loading rate was $10^{7}$ and $10^{8}$ $\mathrm{s}^{-1}$. Identification of twins and dislocations was carried out on the basis of the Common Neighbor Analysis and Dislocation Extraction Algorithm. The visualization of the defective structure of the samples was carried out in the OVITO software [34]. 


\section{Results and discussion}

\subsection{Uniaxial tension}

The simulation results showed that the picture of the origin and development of plasticity under uniaxial tension along the $[11 \overline{2}]$ direction is qualitatively the same for samples with the considered compositions. A change in the loading rate by an order of magnitude also did not affect the features of the structural changes in the crystallites. A typical picture of a defect structure of the crystallite at various values of strain is shown in Fig. 1. The onset of plasticity in simulated crystallites occurs through the twinning. When the elastic limit is reached, a twin emerges in the sample and crosses the crystallite several times due to periodic boundary conditions (Fig. 1a). In the process of the twin propagation the active formation of screw dislocations takes place at its boundaries, which form a dense network between twin plates (Fig. 1b). Upon further stretching, the plastic deformation of the crystallite is provided by the movement and interaction of screw dislocations and the growth of the twin thickness. In this case, the dislocation density, as a rule, decreases, which is clearly seen in Fig. 1c.

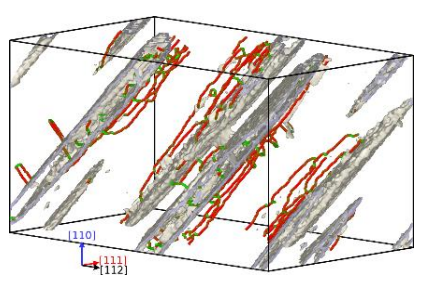

a)

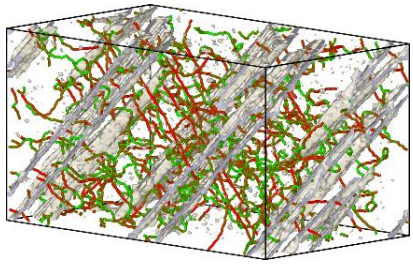

b)

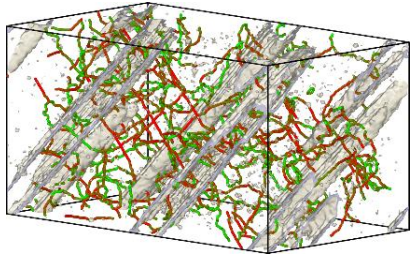

c)

Fig. 1. The defect structure of the V-4Ti crystallite for various stretching values: a) $12.7 \%$, b) $12.8 \%$, c) $15.0 \%$. The gray surfaces are twin boundaries, the red and green lines are screw and edge dislocations. Strain rate is $10^{7} \mathrm{~s}^{-1}$

Analysis of the simulation results shows that using the Metropolis Monte Carlo algorithm to create the initial equilibrium structure rather strongly increases the strength of the simulated samples (samples of the second type). For both concentrations of Ti and both tensile rates, the value of tensile stress at which the twin originates is higher for samples of the second type than for samples of the first type (Fig. 2a). It can be seen from the figure that the stress at the elastic limit of samples of the second type does not depend on the tensile rate. At the same time, this value for samples of the first type substantially depends on the rate of deformation. Such behavior of samples with a random distribution of $\mathrm{Ti}$ atoms is associated with the presence of local regions with a high concentration of $\mathrm{Ti}$, which are stress concentrators and increase the influence of the tensile rate on the nucleation of plasticity. In samples of the second type, as a rule, in the process of plasticity development, a greater number of dislocations form (Fig. 2b), and twin structures occupy a larger volume compared to samples of the first type under similar loading conditions. This may be due to the fact that in the samples of the second type the $\mathrm{Ti}$ atoms are more uniformly distributed over the volume of crystallites. Therefore, plasticity nucleates at higher stresses, which leads to more intense defect formation under loading. 


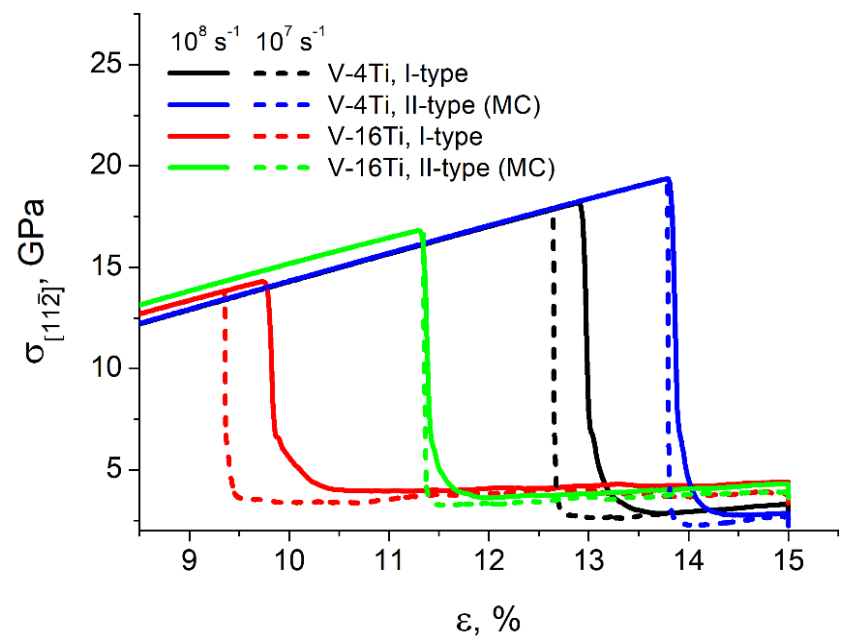

a)

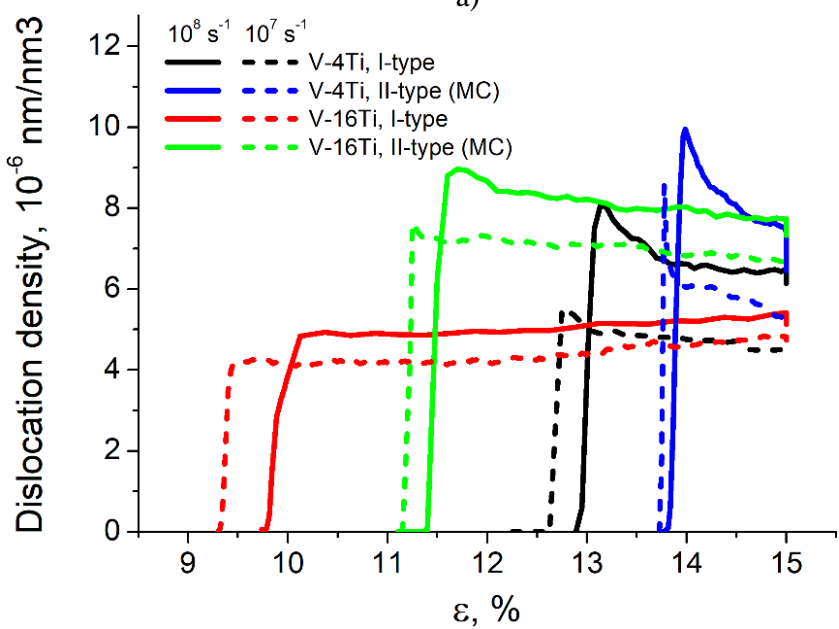

b)

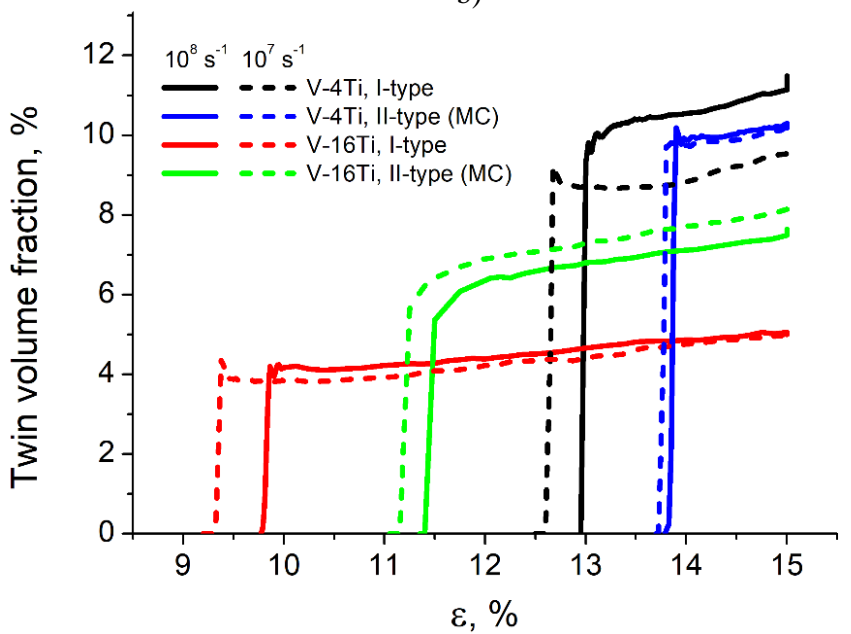

c)

Fig. 2. Dependence of the tensile stress (a), dislocation density (b), the volume fraction of twins (c) on the magnitude of the tensile strain for crystallites of various stoichiometric compositions 
The simulation results show that the elastic limit of the V-Ti system decreases with increasing Ti content. This is consistent with experimental data, which noted that all elastic moduli, except for the shear modulus, decrease with increasing titanium content $[35,36]$.

\subsection{Loading in confined conditions}

A change in the loading scheme significantly altered the characteristics of the onset and development of plasticity in simulated crystallites. Simulation of loading in confined conditions (bilateral compression) was carried out for the samples of the first type. Compared with uniaxial tension, the stresses at the elastic limit for this loading scheme increase significantly. In addition, in the case of the V-4Ti crystallite, the deformation at the elastic limit is almost independent of the deformation rate, while for the V-16Ti crystallite, the loading rate significantly affects this characteristic (Fig. 3a). The weak influence of the strain rate on the onset of plasticity nucleation may be associated with a high stress value at the elastic limit. It is seen in Fig. 2a from the previous section that the effect of the strain rate on the strain value at which defects are generated decreases with increasing stress at the elastic limit. The maximum dislocation density for both concentrations of $\mathrm{Ti}$ increases significantly with increasing strain rate (Fig. 3b). Analysis of the stretched sample structure showed that twins nucleated only in the V-4Ti crystallite, whereas only dislocations were generated in the V-16Ti crystallite. Apparently, this is due to the fact that the stress at the elastic limit for samples with $4 \% \mathrm{Ti}$ concentration is significantly higher than for samples with $16 \%$ Ti concentration (Fig. 3a). To check this assumption, the V-16Ti crystallites of the second type were constructed, in which the distribution of $\mathrm{Ti}$ atoms in the initial structure was obtained using the Metropolis Monte Carlo algorithm. The stress at the elastic limit during the deformation of this sample under constrained conditions was $20 \mathrm{GPa}$. In this sample, along with dislocations, twins began to form.

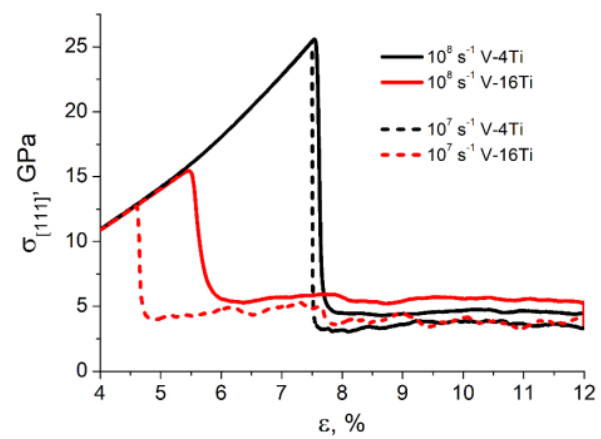

a)

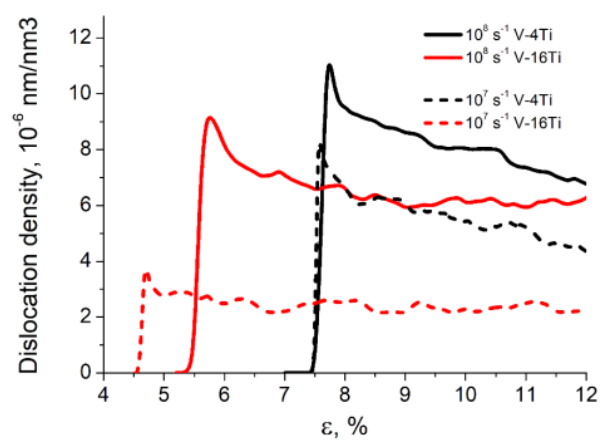

b)

Fig. 3. Dependence of the compressive stress in the [111] direction (a) and dislocation density (b) on the magnitude of the compressive strain for crystallites of various stoichiometric compositions

\section{Conclusions}

Molecular dynamics simulation showed that the nucleation of plastic deformation under uniaxial tension of crystallites $\mathrm{V}-4 \mathrm{Ti}$ and $\mathrm{V}-16 \mathrm{Ti}$ is realized by twinning. Screw dislocations are generated in the stretched crystallites during the growth of twins. An increase in the concentration of Ti leads to a decrease in the strain and stress at which plasticity nucleates in the material. The distribution of Ti atoms in the initial structure has a significant effect on these values. The use of the Metropolis Monte Carlo algorithm makes it possible to redistribute $\mathrm{Ti}$ atoms in the crystallite lattice in such a way that the strength 
characteristics of the material are enhanced and the dependence of the elastic limit on the loading rate $\left(10^{7}-10^{8} \mathrm{~s}^{-1}\right)$ almost disappears. At bilateral compression of $\mathrm{V}-16 \mathrm{Ti}$ samples with a random distribution of titanium, plasticity is generated by the dislocation mechanism, and V-4Ti samples are twinned. This may be due to the low stress at the elastic limit for samples $\mathrm{V}-16 \mathrm{Ti}$, which is not enough to form twins.

The research in the section 3.1 was carried out with financial support of Russian Science Foundation (project №17-19-01374). The research in the section 3.2 was performed under Fundamental Research Program of the State Academies of Sciences for 2013-2020 (line of research III.23)

\section{References}

1. N.A. Polekhina, I.Y. Litovchenko, A.N. Tyumentsev, S.A. Akkuzin, V.M. Chernov, M.V. Leontyeva-Smirnova, Phys. At. Nucl. 81, 1024-1032 (2018)

2. K.V. Almaeva, N.A. Polekhina, I.Y. Litovchenko, A.N. Tyumentsev, V.M. Chernov, M.V. Leont'eva-Smirnova, Russ. Phys. J. 61, 1536-1540 (2018)

3. N.A. Polekhina, I.Y. Litovchenko, A.N. Tyumentsev, D.A. Kravchenko, V.M. Chernov, M.V. Leont'eva-Smirnova, Tech. Phys. 62, 736-740 (2017)

4. T. Muroga, J.M. Chen, V.M. Chernov, R.J. Kurtz, M. Le Flem, J. Nucl. Mater. 455, 263-268 (2014)

5. T. Muroga, "Refractory metals as core materials for Generation IV nuclear reactors," in Structural Materials for Generation IV Nuclear Reactors (Woodhead Publishing, 2017), 415-440.

6. I.Y. Smolin, P.V. Makarov, M.O. Eremin, K.S. Matyko, Procedia Struct. Integr. 2, 3353-3360 (2016)

7. O.I. Cherepanov, I.Y. Smolin, Y.P. Stefanov, P.V. Makarov, Comput. Mater. Sci. 16, 25-31 (1999)

8. V.A. Mikushina, I.Y. Smolin, Y.N. Sidorenko, Key Eng. Mater. 743, 172-175 (2017)

9. A.I. Dmitriev, A.Y. Smolin, V.L. Popov, S.G. Psakhie, Phys. Mesomech. 12, 11-19 (2009)

10. A.I. Dmitriev, A.Y. Smolin, S.G. Psakhie, W. Osterle, H. Kloss, V.L. Popov, Phys. Mesomech. 11, 73-84 (2008)

11. A.Y. Nikonov, A.M. Zharmukhambetova, A.V. Ponomareva, A.I. Dmitriev, Phys. Mesomech. 21, 43-51 (2018)

12. L.A. Zepeda-Ruiz, A. Stukowski, T. Oppelstrup, V.V. Bulatov, Nature 550, 492-495 (2017)

13. A. Shugurov, A. Panin, A. Dmitriev, A. Nikonov, Wear 408-409, 214-221 (2018)

14. D.S. Kryzhevich, K.P. Zolnikov, A.V. Korchuganov, Comput. Mater. Sci. 153, 445-448 (2018)

15. S.G. Psakhie, K.P. Zolnikov, D.S. Kryzhevich, A.V. Korchuganov, Sci. Rep. 9, 3867 (2019)

16. M. Pu, Q. He, J. Zhou, J. Alloys Compd. 795, 241-253 (2019)

17. A.V. Korchuganov, A.N. Tyumentsev, K.P. Zolnikov, I.Yu. Litovchenko, D.S. Kryzhevich, E. Gutmanas, S. Li, Z. Wang, S.G. Psakhie, J. Mater. Sci. Technol. 35, 201-206 (2019)

18. A.V. Korchuganov, K.P. Zolnikov, D.S. Kryzhevich, Mater. Lett. 252, 194-197 (2019) 
19. A.I. Dmitriev, A.Y. Nikonov, A.E. Filippov, V.L. Popov, Phys. Mesomech. 21, 419429 (2018)

20. I.J. Beyerlein, X. Zhang, A. Misra, Annu. Rev. Mater. Res. 44, 329-363 (2014)

21. A.N. Tyumentsev, I.Y. Litovchenko, Y.P. Pinzhin, A.D. Korotaev, S.L. Girsova, V.A. Nesterenkov, Phys. Met. Metallogr. 95, 291-299 (2003)

22. J. Harding, Proc. R. Soc. A Math. Phys. Eng. Sci. 299, 464-490 (1967)

23. K.P. Zolnikov, A.V. Korchuganov, D.S. Kryzhevich, Comput. Mater. Sci. 155, 312-319 (2018)

24. I.F. Golovnev, E.I. Golovneva, V.M. Fomin, Phys. Mesomech. 11, 19-24 (2008)

25. J.R. Greer, J.T.M. De Hosson, Prog. Mater. Sci. 56, 654-724 (2011)

26. M.D. Uchic, P.A. Shade, D.M. Dimiduk, Annu. Rev. Mater. Res. 39, 361-386 (2009)

27. A.I. Dmitriev, A.Y. Nikonov, A.R. Shugurov, A.V. Panin, Appl. Surf. Sci. 471, 318327 (2019)

28. K.P. Zolnikov, A.V. Korchuganov, D.S. Kryzhevich, S.G. Psakhie, Phys. Mesomech. 21, 492-497 (2018)

29. K.P. Zolnikov, D.S. Kryzhevich, A.V. Korchuganov, Lett. Mater. 9, 197-201 (2019)

30. S. Plimpton, J. Comput. Phys. 117, 1-19 (1995)

31. J. Fu, X. Li, B. Johansson, J. Zhao, J. Alloys Compd. 705, 369-375 (2017)

32. A.V. Korchuganov, Math. Montisnigri 42, 65-73 (2018)

33. B. Sadigh, P. Erhart, A. Stukowski, A. Caro, E. Martinez, L. Zepeda-Ruiz, Phys. Rev. B 85, 184203 (2012)

34. A. Stukowski, Model. Simul. Mater. Sci. Eng., 18, 015012 (2010)

35. X. Li et al., Phys. Rev. B 86, 14105 (2012)

36. K.W. Katahara, M.H. Manghnani, E.S. Fisher, J. Phys. F Met. Phys. 9, 773-790 (1979) 